\title{
Histochemical Study of Human Placental Tissues in Gestational Diabetic Mellitus
}

\author{
Israa Ahmed Lafta ${ }^{1}$, Wasan Adnan Abdulhameed ${ }^{1}$, Nahla A. AL-Bakri ${ }^{2}$ \\ ${ }^{1}$ High Institute of Infertility Diagnosis and Assisted Reproductive Technologies, Al Nahrain University, Baghdad, Iraq. \\ israa.ahmmed@ierit.nahrainuniv.edu.iq \\ ${ }^{2}$ College of Education for Pure Sciences, Ibn Al-Haitham, University of Baghdad, Baghdad, Iraq.
}

Gestational diabetes mellitus (GDM) is a serious pregnancy complication in which a woman who has never had diabetes develops chronic hyperglycemia during her pregnancy. Normal placental function is essential for optimal fetal growth. The transport of glucose from the mother to the fetus is critical for fetal nutrient demands and can be stored as glycogen in the placenta. However, the function of this glycogen deposition is unknown: It may well be a source of fuel for a placenta itself or the storage reservoir for the later use by the fetus in times of need. While the significance of the placental glycogen remains unknown, the mounting evidence indicates that the altered glycogen metabolism and/or deposition is associated with many pregnancy complications, such as gestational diabetes, that adversely affect fetal development. The aim of this study is to assess glycogen deposition using Histochemical staining of Periodic Acid Schiff (PAS) stain. The placenta tissue collected from 50 women were enrolled in this study ( 25 women with no complications) and ( 25 women with gestational diabetes). The placentas of the two groups were compared in this study based on glycogen deposition with periodic acid-Schiff stain. The results of a histochemical investigation using PAS stain revealed a significant increase in the glycogen deposition $(\mathrm{p} \leq 0.001)$ in diabetic women's placentas within the intervillous core, around fetal vessels, and the basement membranes.

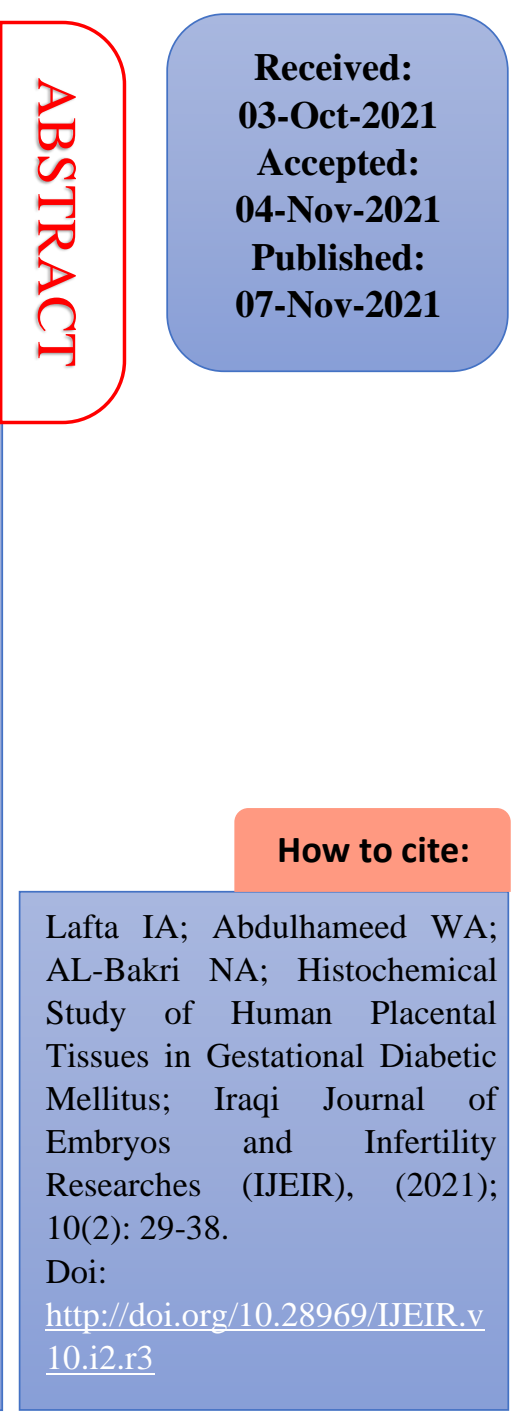

KEYWORDS

Histochemical, Placental, Gestational Diabetic Mellitus 


\section{Introduction}

Gestational Diabetes Mellitus (GDM) is the most common metabolic disorder that found during gestation and is define as hyperglycemia of variable severity with onset or first recognition during gestation that does not clearly characterize any form of the preexisting diabetes (American Diabetes Association ${ }^{[1]}$ ). It affects approximately $16.5 \%$ of pregnancies worldwide (Plows, et al. $\left.{ }^{[2]}\right)$. The placenta is an organ that connects the mother and her fetus during pregnancy (Gul, et al. $\left.{ }^{[3]}\right)$. In the placenta, glucose can be transformed into glycogen for storage by either glycogen synthase or using glycogenin as a prime. However, the function of glycogen deposition stays a matter of debate, it may be the source of fuel for placenta itself or the storage pool for the later use by fetus in the times of need, while the importance of the placental glycogen stays elusive. Increasing evidence indicates that the changed glycogen metabolism and the deposition accompanies with numerous pregnancy complications that harmfully affects fetal development specially diabetes mellitus (Akison, et al. ${ }^{[4]}$ ).

\section{Materials and Methods}

The placental sample was collected from 50 pregnant women that were included in this study from the Department of Obstetrics and Gynecology in Al Yarmouk Teaching Hospital, Baghdad, Iraq. The written informed consent was obtained from each woman, the first group include 25 uncomplicated pregnant women and the second groups included 25 gestational diabetes pregnant women. The pathological examination was performed according to the methods of (Suvarna, et al. ${ }^{[5]}$, Mahmoud, et al. $\left.{ }^{[6]}\right)$, after prior fixation of the placental fragments in formaldehyde, washing, dehydration and embedding in paraffin block (hot paraffination), followed by the cooling. A paraffin block was cut by the rotary microtome, it spread on the blade, then it was introduced in the thermostat for deparaffination, followed by a staining procedure with the Periodic Acid SchiffHematoxylin (PAS-H) staining.

\section{Results}

The Image $\mathbf{J}$ software was used to evaluate the quantification of Periodic Acid Schiff (PAS) 
stain in sections of the control and gestational diabetic mellitus groups. In the control group Table 1, 15(60.00\%) cases displayed the hazy reaction ranged from (7.07-18.50\%) of the area stained with the red-magenta color Figure 1 and 10(40.00\%) cases registered the traces reaction ranged from (20.10-37.10\%) of area stained with the red-magenta color. While, in the gestational diabetic mellitus group Table 1, $4(16.00 \%)$ cases were having the traces PAS positive ranged from (21.60-30.9\%) of the area stained with the red-magenta color and $4(16.00 \%)$ cases were having a mild reaction ranged from (40.50-42.70\%) of the area stained with the red-magenta color. As well as $11(44.00 \%)$ were having the moderate PAS positive ranged from (63.20-79.80) of the area stained with the red-magenta color. Although $6(24.00 \%)$ cases recorded the strong PAS positive ranged from $(81.20-89.50 \%)$ of the area stained with the red-magenta color Table 1. Therefore, gestational diabetic mellitus group had a higher strong PAS positive and a higher moderate and mild PAS positively. Likewise, the control group did not record any cases in any of the three mentioned scoring categories, and the majority of cases in this group had hazy or trace reactions. Statistical analysis revealed that the difference in cases percentage of PAS staining score was highly significant $(\mathrm{p} \leq 0.001)$ between the two groups with all scoring as shown in Figure 2 and Figure 3. On the other hand, the current study found an increase in the expression of PAS stain in term of gestational diabetes mellitus placenta $(63.6 \pm 4.7)$ compared with the preterm of gestational diabetes mellitus placenta $(42.3 \pm 16.4)$, as shown in Table 2 .

\section{Discussion}

Diabetes is associated with lower glycogen levels in maternal peripheral tissues as a result of diabetes-related insulin resistance; however, the placenta is the only organ in which diabetic pregnancies have higher glycogen levels than non-diabetic pregnancies (Desoye, et al. ${ }^{[7]}$ ). Reduced fetal-placental blood flow may compensate for increased fetal glucose supply, and increased glucose storage as glycogen in the placenta may contribute to some fetal safety, but only to a limited extent (Desoye, et al. ${ }^{[8]}$, Akison, et al. ${ }^{[4]}$ ). 
Table (1): Periodic Acid Schiff reactivity in section placental of control and gestational diabetic mellitus by using digital quantification of Periodic Acid Schiff staining

\begin{tabular}{|c|c|c|c|}
\hline Scoring & $\begin{array}{c}\text { Control }(\mathrm{N}=25)(\%) \\
\text { (Range) }\end{array}$ & $\begin{array}{c}\text { GDM }(\mathbf{N}=25)(\%) \\
\text { (Range) }\end{array}$ & p-value \\
\hline Hazy $(<20 \%)$ & $\begin{array}{c}15(60.00) \\
(7.07-18.50)\end{array}$ & $0(0)$ & $\begin{array}{c}0.000 * \\
*\end{array}$ \\
\hline $\begin{array}{c}\text { Traces (20- } \\
39.99 \%)\end{array}$ & $\begin{array}{c}10(40.00) \\
(20.10-37.10)\end{array}$ & $\begin{array}{c}4(16.00) \\
(21.60-30.9)\end{array}$ & $\begin{array}{c}0.000 * \\
*\end{array}$ \\
\hline $\begin{array}{l}\text { Mild (40- } \\
59.99 \%)\end{array}$ & $0(0.00)$ & $\begin{array}{c}4(16.00) \\
(40.50-42.70)\end{array}$ & $\begin{array}{c}0.000^{*} \\
*\end{array}$ \\
\hline $\begin{array}{c}\text { Moderate (60- } \\
\mathbf{7 9 . 9 9 \% )}\end{array}$ & $0(0.00)$ & $\begin{array}{c}11(44) \\
(63.20-79.80)\end{array}$ & $\begin{array}{c}0.000 * \\
*\end{array}$ \\
\hline Strong $(>80 \%)$ & $0(0.00)$ & $\begin{array}{c}6(24.00) \\
(81.20-89.50)\end{array}$ & $\begin{array}{c}0.000^{*} \\
*\end{array}$ \\
\hline
\end{tabular}

(2-tailed) independent t-test; * highly significant at $p \leq 0.001$.

Table (2): Expression of periodic acid Schiff stain in term and preterm of gestational diabetes mellitus placenta

\begin{tabular}{|c|c|c|c|}
\hline PAS stain & $\begin{array}{c}\text { Preterm } \\
(\text { Mean } \pm \text { SEM) }\end{array}$ & $\begin{array}{c}\text { Term } \\
(\text { Mean } \pm \text { SEM) }\end{array}$ & P-Value \\
\hline $\begin{array}{c}\text { Level of periodic acid } \\
\text { Schiff stain }\end{array}$ & $42.3+16.4$ & $63.6+4.7$ & $0.05^{*}$ \\
\hline
\end{tabular}

(2-tailed) independent t-test; * significant at $p \leq 0.05$. 


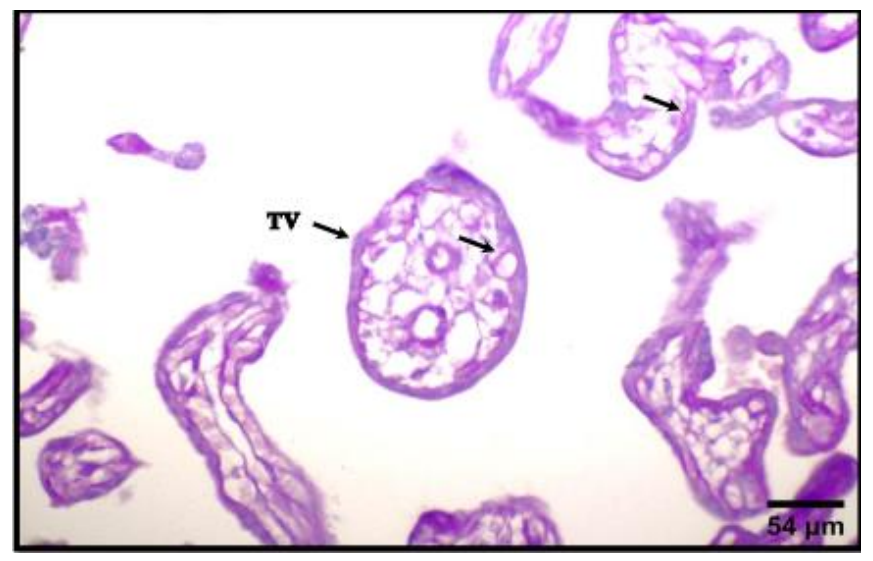

Figure (1): Control placenta showing: Hazy reactive, areas of glycogen deposition (arrow); terminal villous (TV) (PAS stain, 40X)

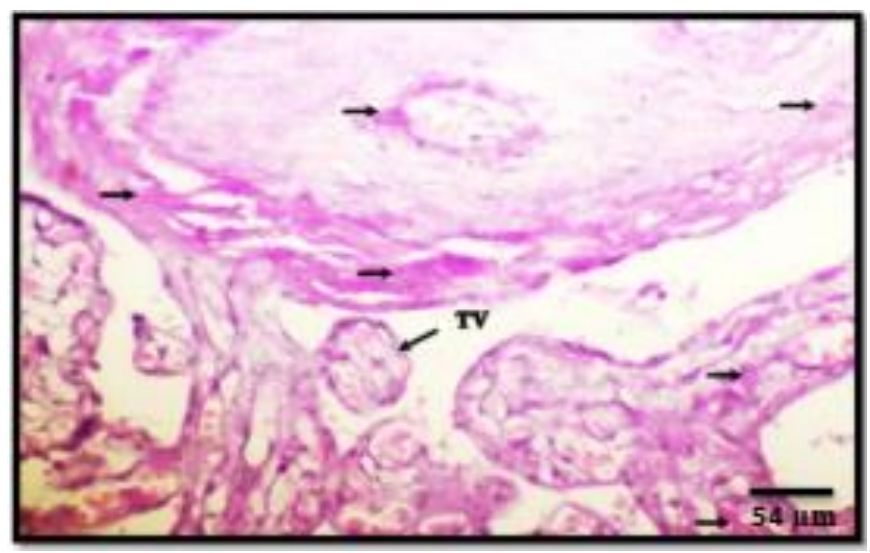

Figure (2): GDM placenta showing: moderate reactive, areas of glycogen deposition (arrow) (PAS stain ,40X)

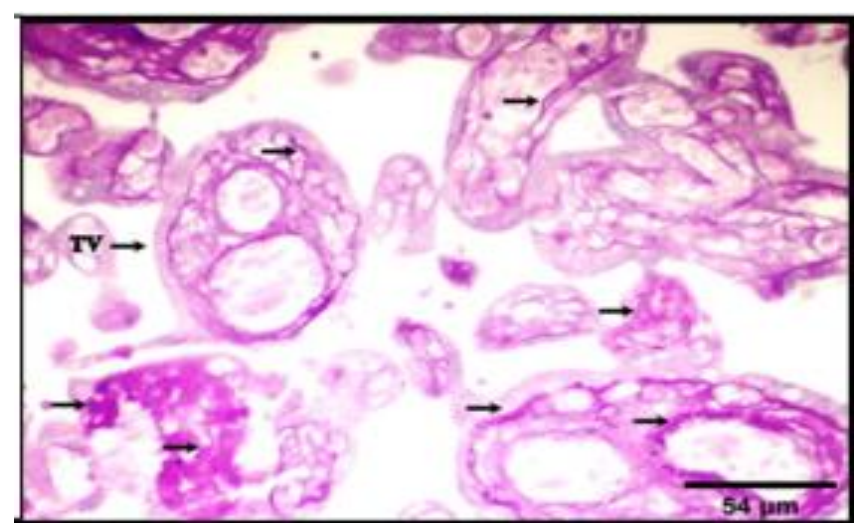

Figure (3): GDM placenta showing: strong reactive, areas of glycogen deposition (arrow); terminal villous (TV) (PAS stain, 40X). 
Periodic Acid Schiff stain was used in this study to detect the amount of the glycogen in the tissue samples, and was quantified using the Image $\mathbf{J}$ software. The software has been used in numerous studies to quantify PAS staining in animal placentas (Rampon, et al. ${ }^{[9]}$, He, et al. $\left.{ }^{[10]}\right)$. The current study classified PAS staining data into the five categories (Hazy, Traces, Mild, Moderate and Strong). This description is similar to the previous literature that have provided a descriptive analysis of PAS staining of placenta with diabetics (Tewari, et al. ${ }^{[11]}$, Nidhi SK ${ }^{[12]}$ ). The current study, showed PAS reactivity for the glycogen in tissue sections was much stronger in the gestational diabetic mellitus group compared to control group, which showed higher level in hazy reactivity, followed by a trace's reactivity but not a mild, moderate and strong reactions. It is mainly concentrated around the fetal blood vessel and around the villi. GDM placentae had the highest moderate positive and the strong reactivity, followed by the mild and trace reactions in some females that took insulin. As a result of this study in agreement, founded that the insulin-treated women have similar glycogen content to the healthy women (Desoye, et al. ${ }^{[7]}$ ). Furthermore, women undergoing the diet-intervention have only the slightly lower placental glycogen levels, which may be related to insulin receptor expression in trophoblast. In this study, PAS-stained sections revealed the highly concentrated magenta red staining area in GDM groups at the intervillous core, around fetal vessels, and in basement membranes of trophoblastic cells, which cover a terminal villi. There is an increased deposition of glycogen in GDM placenta are a common feature, mainly around fetoplacental vessels, the syncytiotrophoblast cells and villous stroma (Martino, et al. ${ }^{[13]}$ ). According to (Fard, et al. ${ }^{[14]}$, Treesh SA ${ }^{[15]}$ ), who used PAS stain to examine placentae diabetic mellitus, gestational diabetic mellitus and displayed a marked thickening of basement membrane in a diabetic placentae and the glycogen was deposit in the cells surrounding fetoplacental vasculature as well as within stromal cell of the terminal villous in comparison of the control groups. This finding 
is consistent with the findings of the current study.

\section{Conclusions}

In conclusion, the Periodic Acid Schiff stain was much stronger in the Gestational Diabetes Mellitus group compared to the control group. It is mainly concentrated at the intervillous core, around the fetal vessels, and basement membranes of the trophoblastic cells, this is an indication that the highest glycogen content is found in diabetic pregnant women's placenta. In addition, the glycogen deposition in the term placenta were found to be higher than the preterm placenta of gestational diabetes mellitus group.

\section{Acknowledgment}

We would like to acknowledge Al Nahrain University, Baghdad, Iraq.

\section{Funding}

This work received no funding.

\section{Author Contribution}

Lafta, IA, performed the study, examined and reviewed results, and manuscript writing with the help and supervision of Abdulhameed, WA, and AL-Bakri, NA.

\section{Conflict of Interest}

The authors declare no conflict of interest.

\section{Ethical Clearance}

The study was approved by the Ethical Approval Committee.

\section{References}

[1]American Diabetes Association. 2. Classification and Diagnosis of Diabetes. Diabetes Care. American Diabetes Association; 2016 Dec 15;40 (Supplement 1):S11-S24. [Online article link]

[2]Plows J, Stanley J, Baker P, Reynolds C, Vickers M. The Pathophysiology of Gestational Diabetes Mellitus. International Journal of Molecular Sciences. MDPI AG; 2018 Oct 26;19(11):3342. [Online article link]

[3] Gul M, Bayat N, Cetin A, Kepekci RA, Simsek Y, Kayhan B, et al. Histopathological, Ultrastructural and Apoptotic Changes in Diabetic Rat Placenta. 
Balkan Medical Journal. AVES Publishing Co.; 2015 Jul 6;32(3):296-302. [Online article link]

[4] Akison LK, Nitert MD, Clifton VL, Moritz KM, Simmons DG. Review: Alterations in placental glycogen deposition in complicated pregnancies: Current preclinical and clinical evidence. Placenta. Elsevier BV; 2017 Jun;54:52-8. [Online article link]

[5] Suvarna KS, Layton C, Bancroft JD (Eds.). Bancroft's theory and practice of histological techniques E-Book. Elsevier Health Sciences, 2018. [Online article link]

[6] Mahmoud EA, Al-Bakri NA, Qasim BJ. Histomorphometrical Study of Placental from Male and Female Neonates of Diabetic Women. Ibn AL- Haitham Journal For Pure and Applied Science. College of Education for Pure Sciences Ibn Al-Haitham; 2019 May 20;32(2):1. [Online article link]

[7]Desoye G, Hofmann HH, Weiss PAM. Insulin binding to trophoblast plasma membranes and placental glycogen content in well-controlled gestational diabetic women treated with diet or insulin, in well- controlled overt diabetic patients and in healthy control subjects. Diabetologia. Springer Science and Business Media LLC; 1992 Jan;35(1):45-55. [Online article link]

[8] Desoye G, Shafrir E, Hauguel-de Mouzon S. The placenta in diabetic pregnancy: placental transfer of nutrients. Textbook of Diabetes in Pregnancy. 2nd ed. informa healthcare, London. 2008;47-56. [Online article link]

[9] Rampon C, Bouillot S, Climescu-Haulica A, Prandini M-H, Cand F, Vandenbrouck Y, et al. Protocadherin 12 deficiency alters morphogenesis and transcriptional profile of the placenta. Physiological Genomics. American Physiological Society; 2008 Jul;34(2):193-204. [Online article link]

[10] He N, Lim SJ, Moreira de Mello JC, Navarro I, Bialecka M, Salvatori DCF, et al. At Term, $\mathrm{XmO}$ and $\mathrm{XpO}$ Mouse Placentas Show Differences in Glucose Metabolism in the Trophectoderm-Derived Outer Zone. Frontiers in Cell and Developmental Biology. Frontiers Media SA; 2017 Jun 21;5. [Online article link] 
[11] Tewari V, Tewari A, Bhardwaj N. Histological and histochemical changes in placenta of diabetic pregnant females and its comparision with normal placenta. Asian Pacific Journal of Tropical Disease. Elsevier BV; 2011 Mar;1(1):1-4. [Online article $\underline{\text { link] }}$

[12] Nidhi Santhosh Kumar M. Gross Morphological and Pathological Changes in Placenta of Diabetic Patients and Its Association with Fetal Outcome. Stanley Medical College, Chennai; 2016. [Online article link]

[13] Martino J, Sebert S, Segura MT, GarcíaValdés L, Florido J, Padilla MC, et al. Maternal Body Weight and Gestational Diabetes Differentially Influence Placental and Pregnancy Outcomes. The Journal of Clinical Endocrinology \& Metabolism. The Endocrine Society; 2016 Jan 1;101(1):5968. [Online article link]

[14] Fard HMA, Afshari F, Samani ZG. Histological Study of the Placenta in Neonates With Intrauterine Growth Retardation. Crescent Journal of Medical and Biological Sciences. 2016 Apr;3(3):107-110. [Online article link]

[15] Treesh SA. Histological Changes of the Human Placenta in Pregnancies Complicated with Diabetes. Journal of Cytology \& Histology. OMICS Publishing Group; 2015;06(02). [Online article link]

\section{Peer Review Information}

Double-Blind Peer Review in which both authors and reviewers does not know each other.

This work was reviewed by

Asst. Prof. Dr. Almagul M Kurmanova

Asst. Prof. Dr. Mostafa A. Mousa

\section{Editorial Policy}

The editorial policy at IJEIR ensured that this article fit the standards of scientific publications.

This work was copyedited by

Dr. Taif Alawsi 


\section{Authors Biographies}

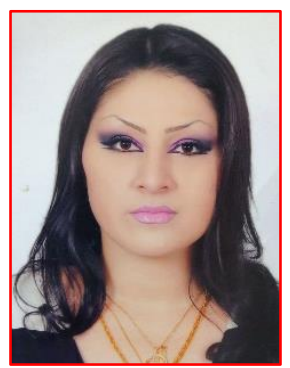

\section{Israa Ahmed Lafta}

She received her B.Sc. in Biology from the College of Science, Al Mustansiriya University, in 2008. She worked in the laboratories of the Iraqi Ministry of Health. She received her M.Sc. in applied embryology in 2021 from the High Institute of Infertility Diagnosis and Assisted Reproductive Technologies, Al Nahrain University, Baghdad, Iraq.

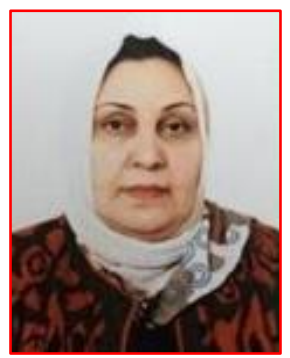

\section{Dr. Wasan Adnan Abdulhameed}

She received M.B.CH.B. from the University of Baghdad, College of medicine in 1992, the diploma and the Arab Board in Gyn. and Obs. from Medical City, Baghdad in 1998. She worked at Al Sharjah University, UAE. She earned a laparoscopic surgery certificate from India in 2009. She was a Lactation consultant in Australia in 2010. She occupied many administrative positions She attended more than 20 academic congresses internationally.

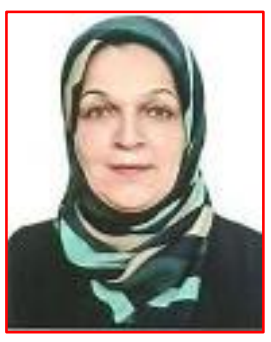

\section{Dr. Nahla A. AL-Bakri}

She received the B.Sc., M.Sc., and Ph.D. in Biology, from the College of Science, the University of Baghdad in 1982, 1987, and 1996, respectively. She is a professor of biology. She worked in the Baghdad University, College of Education for Pure Science (Ibn AlHaitham), Department of Biology. She occupied many administrative positions, and the Number of Publications
Researches: 70, Number of Letters of Appreciation and Certificates of Appreciation: 80, Books Authored In Arabic: 2, and the Number of Conferences in which she participated in Iraq: 29, and in International Conferences: 13.

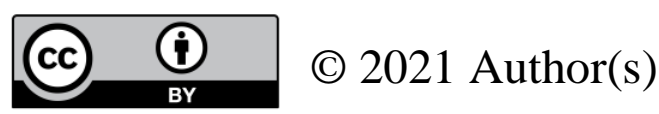

This article is licensed under a Creative Commons Attribution 4.0 International License, which permits use, sharing, adaptation, distribution and reproduction in any medium or format, as long as you give appropriate credit to the original author(s) and the source, provide a link to the Creative Commons license, and indicate if changes were made.

\section{http://creativecommons.org/licenses/by/4.0/.}

